\title{
Mosaic Turner syndrome and pituitary microadenoma in patient with polyglandular autoimmune
}

syndrome type II

\section{O. Boskovic, S.Medenica}

Clinical Center of Montenegro

\section{Introduction}

Polyglandular autoimmune syndrome type II (PGA-II) is the most common immunoendcrinopathy syndrome, characterized by the obligatory occurrence of Addison disease in combination with thyroid autoimmune diseases and/or type 1 diabetes mellitus. This case report presents coexistence of mosaic Turner syndrome and pituitary micoradenoma in patient with PGA-II.

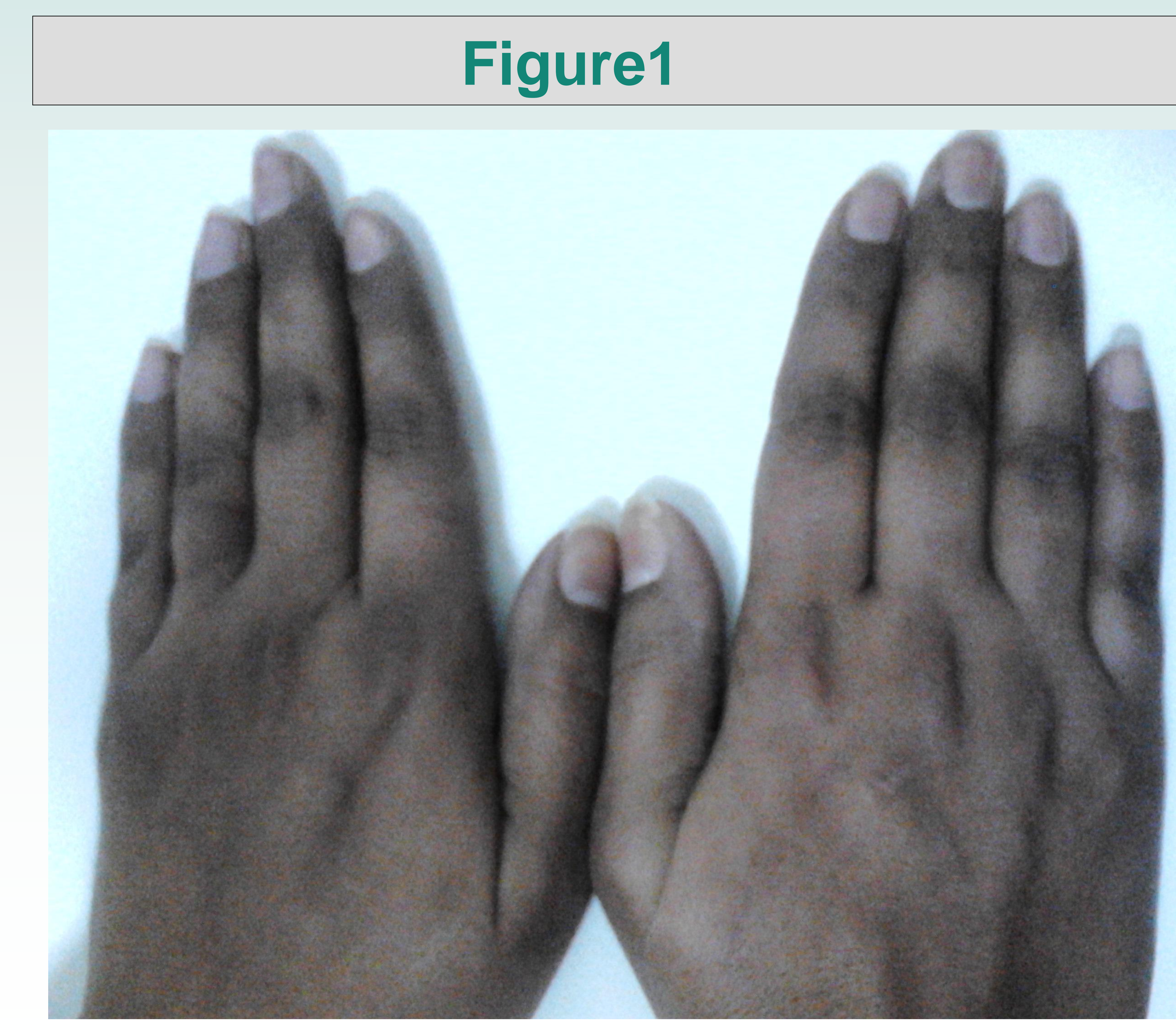

\section{Case report}

A 30 year old women underwent in vitro fertilization (IVF) for four times, with no success (always poor ovarian response, double embryo transfer). Hashimoto thyroiditis and subclinica hypothyreoidismus were diagnosed. Levothyroxine substitution was started. Repeatedly elevated prolactin level in the morning, but preserved circadian rhythm and daily values in the referent range. NMR sellar region shown microadenoma in the right half. No adequate cortisol answer in insulin tolerance test, but normal prolactine and growth hromone. In thyrotropin relasing hormone test no paradoxal response. Husband has the oligoasthenospermia, the varicocele surgically removed and normal karyotype (46, XY). Patient's karyotype testing shown a mosaic monosomy $X$ $(46, X X / 45, X 0)$, with $5 \%$ of analyzed cells caracterized by monosomy $X$, but no syndrome fenotype characteristics, entered puberty at the time, regular menstrual cycles and echocardiography dysgenetic ovarian caracteristics. During the last IVF no follicle on folitropine stimulation was found, and for the first time higher $\mathrm{FSH}$ value and lower $\mathrm{AMH}$ value were found, indicating premature ovarian insuffuciency; antiovarian antibodies were negative exluding immune-mediated process. A year later, menstrual cycles became irregular.

\section{Case report}

Two years later she presented with sings of hypocorticism- artralgia, hyperpigmentation, fatigue, hypotension and low corisol level, but normal electrolyte level, hydrocortisone substitution was started (Figure 1,2). PTH was in referent range. Positive anti GAD and ant IA2 antibodies were demonstrated, oral glucose tolerance test was normal. Patient is now in oocyte donation process.

\section{Conclusion}

Oocyte donation may offer solution to women with multiple autoimmune disorder causing infertility.

\section{Figure 2.}

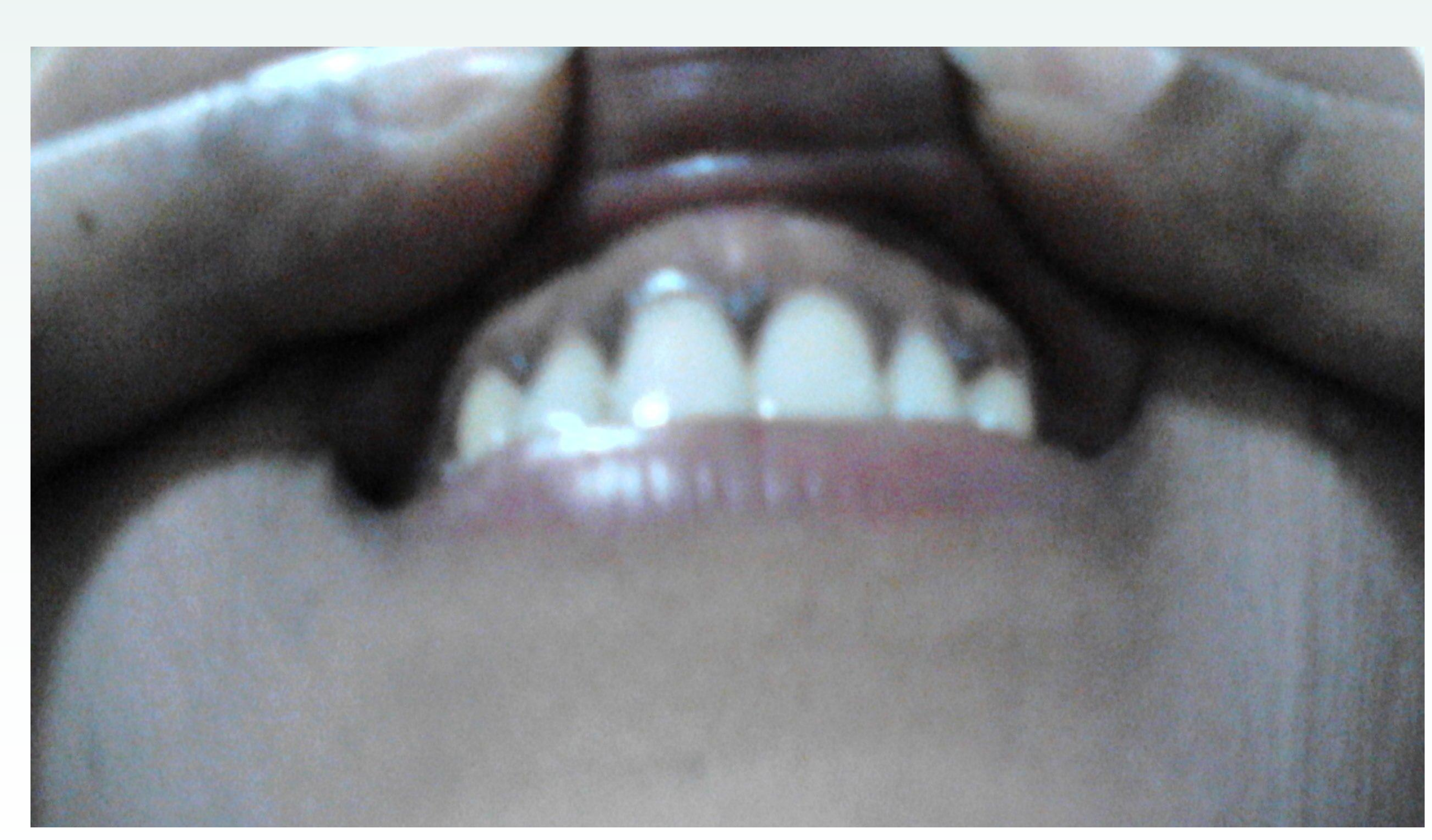

